\title{
Reduced adherence to antiretroviral therapy is associated with residual low-level viremia
}

This article was published in the following Dove Press journal:

Pragmatic and Observational Research

26 May 2017

Number of times this article has been viewed

\author{
Franco Maggiolo' \\ Elisa Di Filippo' \\ Laura Comi' \\ Annapaola Callegaro ${ }^{2}$ \\ Giorgio L Colombo ${ }^{3,4}$ \\ Sergio Di Matteo ${ }^{4}$ \\ Daniela Valsecchi ${ }^{5}$ \\ Marco Rizzi' \\ 'USC of Infectious Diseases, \\ 'USC Microbiology and Virology, \\ ${ }^{3}$ Department of Drug Sciences, \\ University of Pavia, ${ }^{4}$ S.A.V.E. Studi - \\ Health Economics \& Outcomes \\ Research, Milan, ${ }^{5}$ USC of Pharmacy, \\ ASST Papa Giovanni XXIII, \\ Bergamo, Italy
}

\begin{abstract}
The source and significance of residual low-level viremia (LLV) during combinational antiretroviral therapy (cART) remain a matter of controversy. It is unclear whether residual viremia depends on ongoing release of HIV from the latent reservoir or if viral replication contributes to LLV. We examined the relationship between adherence and LLV. Adherence was estimated by pharmacy refill and dichotomized as $\geq 95 \%$ or $<95 \%$. Plasma HIV-RNA was determined, with an ultrasensitive test having a limit of detection of 3 copies $/ \mathrm{mL}$ at least 2 times over the follow-up period. Patients were grouped according to HIV-RNA over time as $\mathrm{K}<3$ : constantly $<3$ copies $/ \mathrm{mL}$; $\mathrm{V}<3$ : sometimes below or above the cutoff limit but always $<50$ copies $/ \mathrm{mL} ; \mathrm{K}>3$ : constantly between 3 and 50 copies $/ \mathrm{mL}$; and $V>50$ : a measure of $>50$ copies $/ \mathrm{mL}$ minimum. Overall, 2789 patients were included. At each time point approximately $92 \%$ of the patients presented an HIV-RNA $<50$ copies $/ \mathrm{mL}$ and two-thirds of those $<3$ copies $/ \mathrm{mL}, 34.6 \%$ of patients had $<3$ copies $/ \mathrm{mL}$ constantly, $32.7 \%$ sometimes below or above the cutoff limit but always $<50$ copies $/ \mathrm{mL}, 9.5 \%$ constantly between 3 and 50 copies $/ \mathrm{mL}$, and $23.2 \%$ a measure of $>50$ copies $/ \mathrm{mL}$ minimum. The mean adherence rate was $92.1 \%(95 \%$ confidence interval [CI] from $91.1 \%$ to $93.1 \%)$ in $\mathrm{K}<3$ patients, similar in $\mathrm{V}<3$ patients $(91.9 \%)$, but lowered to $88.8 \%$ in $\mathrm{K}>3$ patients and to $88.4 \%$ in $\mathrm{V}>50$ patients $(P<0.0001)$. Approximately $55 \%$ of patients in groups $\mathrm{K}<3$ and $\mathrm{V}<3$ showed an adherence rate $\geq 95 \%$; this proportion lowered to $\sim 51 \%$ in $\mathrm{K}>3$ and to $48 \%$ in $\mathrm{V}>50$. Moreover, $34 \%$ of patients with a steady adherence $<95 \%$ were categorized as $\mathrm{K}>3$, whereas $21.7 \%$ of those with a drug holiday $(21.7 \%$ ) were observed in the $\mathrm{V}>50$ group $(P=0.002)$. A steady viral suppression can occur despite moderate cART non-adherence, but reduced adherence is associated with low-level residual viremia, which could reflect new rounds of HIV replication. However, a detectable HIV-RNA could also be detected in patients with optimal cART adherence, indicating additional mechanisms favoring HIV persistence.
\end{abstract}

Keywords: adherence, drug-refill, LLV, low-level viremia, viral replication, residual viremia cART

\section{Introduction}

The development of antiretroviral (ARV) therapy (combinational antiretroviral therapy [cART]) has drastically reduced HIV/AIDS-associated morbidity and mortality worldwide. ${ }^{1}$ Adherence to cART is the major factor influencing the extent and durability of optimal treatment response, including reaching and maintaining non-detectable plasma HIV-RNA levels. ${ }^{2,3}$

The quantification of HIV-RNA in plasma is an essential tool for monitoring the efficacy of cART because of the observation that suppression of viral replication predicts immunological and clinical outcomes in HIV-positive patients. Achieving and maintaining viral load "undetectability" is the recommended target of cART in
Correspondence: Franco Maggiolo USC Malattie Infettive, ASST Papa Giovanni XXIII, Via Oms I, 24I28, Bergamo, Italy

Email franco31556@hotmail.com 
all international guidelines. ${ }^{4,5}$ After the introduction of HIVRNA monitoring in clinical practice in 1996, the target goal to define viral suppression has been defined by the technical limits of available assays rather than by biological considerations. For first-generation tests, it was 400-500 copies/ $\mathrm{mL}$, and then lowered to $50-75$ copies $/ \mathrm{mL}$ with secondgeneration tests.

In recent years, new commercial assays have been introduced based on real-time polymerase chain reaction that have a lower limit of quantification of 20 or 40 copies $/ \mathrm{mL}$ and can also report qualitative/quantitative RNA detection below these thresholds. ${ }^{6}$

Although these assays have now entered routine practice, the source and significance of this "residual" viremia during cART remain a matter of controversy. ${ }^{7}$ In particular, it is unclear whether the residual viremia $<50$ copies $/ \mathrm{mL}$ should be regarded as an indication to review drug potency or pharmacokinetic interactions, tolerability of the regimen, adherence to prescribed doses, or selection of drug-resistant viruses.

We, therefore, conducted a study in our cohort specifically examining the relationship between adherence and residual low-level viremia (LLV).

\section{Materials and methods}

This is a single-center, cohort study. As in previous studies, we estimated $\mathrm{ARV}$ adherence using a validated measure based on pharmacy refill records. ${ }^{89}$ The Italian Health Care System delivers all HIV care including medications at no charge and uses a computerized tool to capture every instance of $\mathrm{ARV}$ dispensation in a centralized registry. These data were cross-linked with the clinical electronic database that allows to obtain a comprehensive retrospective and prospective clinical profile for all participants including, but not limited to, demographic characteristics, number and type cART regimens, total duration of cART exposure, HIV-RNA levels and CD4 cell counts over time, co-medications, and clinical events. All patients gave their written informed consent for their data to be stored in this database and for the use of data in an aggregated form for study and scientific purposes. For retrospective studies of purely observational nature with no intervention applied on patients (e.g., database extraction of data), the Italian law requires that the relevant Ethics Committee is notified of the study, but do not have to deliver a formal approval. The Ethics Committee of Papa Giovanni Hospital was notified of the study and approval was deemed not necessary.

The present analyses considered all HIV-positive patients who had been dispensed at least 1 day of cART between October 1, 2012, and September 30, 2014.
Adherence to cART was the primary outcome in this study. Adherence was estimated by calculating the number of days for which an individual was dispensed cART over the number of days of effective follow-up resulting in the percentage of time the patient was without medication over the considered time interval. For patients still on therapy at the end of the observational period, the follow-up was censored at the date of last drug pickup.

We dichotomized adherence measure as $\geq 95 \%$ or $<95 \%$ as this cutoff level has previously been shown to be significantly associated with virological suppression. ${ }^{10}$ As in our center, HIV drugs are dispensed as 60 day pickups, we calculated adherence on a bimonthly basis, too. For patients with adherence $<95 \%$, we compared overall adherence with adherence calculated by the sum of single 60 day refills and defined the presence of a drug holiday as a discrepancy $>10 \%$ between these two measures (being the sum of adherence rates between single pickups greater).

Plasma HIV-RNA levels were determined with an ultrasensitive, previously validated HIV-RNA test based on a real-time polymerase chain reaction having a limit of detection of 3 copies $/ \mathrm{mL} .^{11}$

Patients were grouped according to their virological response over time. The first group included subjects with HIV-RNA over time as constantly $<3$ copies/mL $(\mathrm{K}<3)$; the second group included subjects sometimes below or above the cutoff limit but always $<50$ copies $/ \mathrm{mL}(\mathrm{V}<3)$; the third group included subjects constantly between 3 and 50 copies $/ \mathrm{mL}$ $(\mathrm{K}>3)$; and the fourth group included subjects with a measure of $>50$ copies $/ \mathrm{mL}$ minimum $(\mathrm{V}>50)$.

Descriptive results are presented as proportions, means, standard deviations, and 95\% confidence intervals (CIs). Inferential statistics, in unadjusted assessment, were based on Fisher's exact test for categorical variables and the analysis of variance test for continuous variables. The associations between residual LLV and factors such as cART adherence were performed using Spearman or Wilcoxon tests. Adjusted logistic regression was used for multivariable analysis. The multivariable models included residual viremia, as previously defined, as a dependent variable, as well as variables significantly associated in unadjusted analysis.

\section{Results}

Overall, 2789 HIV-positive patients were included in the study (Figure 1); 74\% $(n=1916)$ were men, and their mean age was 46.5 years (SD, 9.5). The vast majority $(88.1 \%)$ were of Caucasian heritage. Heterosexual contact (47.7\%) was the most common risk factor for HIV followed by intravenous drug use (30.7\%), homosexual contacts (19.5\%), and other 


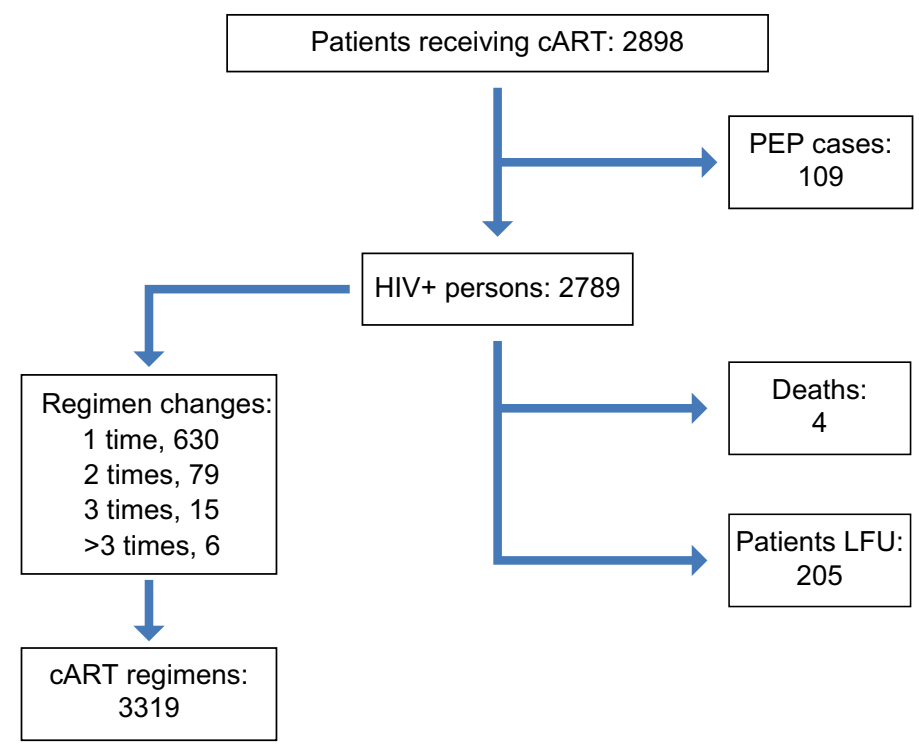

Figure I Patient flow chart.

Abbreviations: CART, antiretroviral therapy; LFU, lost to follow-up; PEP, post exposure prophylaxis.

factors (2.1\%). A HCV co-infection was diagnosed in $28.3 \%$ of patients, whereas HBV co-infection was present in 5.1\% of them. Baseline pre-cART mean HIV-RNA was $5.33 \mathrm{log}$ copies $/ \mathrm{mL}$ (SD, 5.86). The mean number of years on cART was 8.9 (SD, 6.3), whereas the mean duration of current cART was 2.2 years (SD, 3.2). Patients had experienced a mean of 4 cART lines (SD, 3.2). Most of the patients ( $86 \%$ ) were on a triple-drug combination, while $12.2 \%$ took a simplified two-drug regimen (Figure 2). The most common nucleas $(\mathrm{t})$ ide reverse transcriptase inhibitor (NRTI) was Viread (tenofovir; 64.9\%) followed by Ziagen (abacavir; 12.8\%). Forty-seven percent of patients were on non-nucleoside reverse transcriptase inhibitor
(NNRTI) regimens, $29.7 \%$ on boosted protease inhibitor (PI) regimens, and $11.3 \%$ on integrase inhibitor (INI) containing regimens. However, drug class use was not evenly distributed as NNRTIs were more frequently prescribed in first lines of cART, whereas PIs and INIs were often a part of subsequent regimens $(P<0.0001)$. Virological response to cART was stable over time. About $92 \%$ of the patients presented, at any time, an HIV-RNA $<50$ copies/mL (Figure 3 ) and two-thirds of them presented an HIV-RNA $<3$ copies $/ \mathrm{mL}$. However, only $34.6 \%$ of patients steadily had a viral load $<3$ copies/ $\mathrm{mL}(\mathrm{K}<3)$, whereas $32.7 \%$ could be categorized as $\mathrm{V}<3$ and $9.5 \%$ and $23.2 \%$ as $\mathrm{K}>3$ and $\mathrm{V}>50$, respectively.

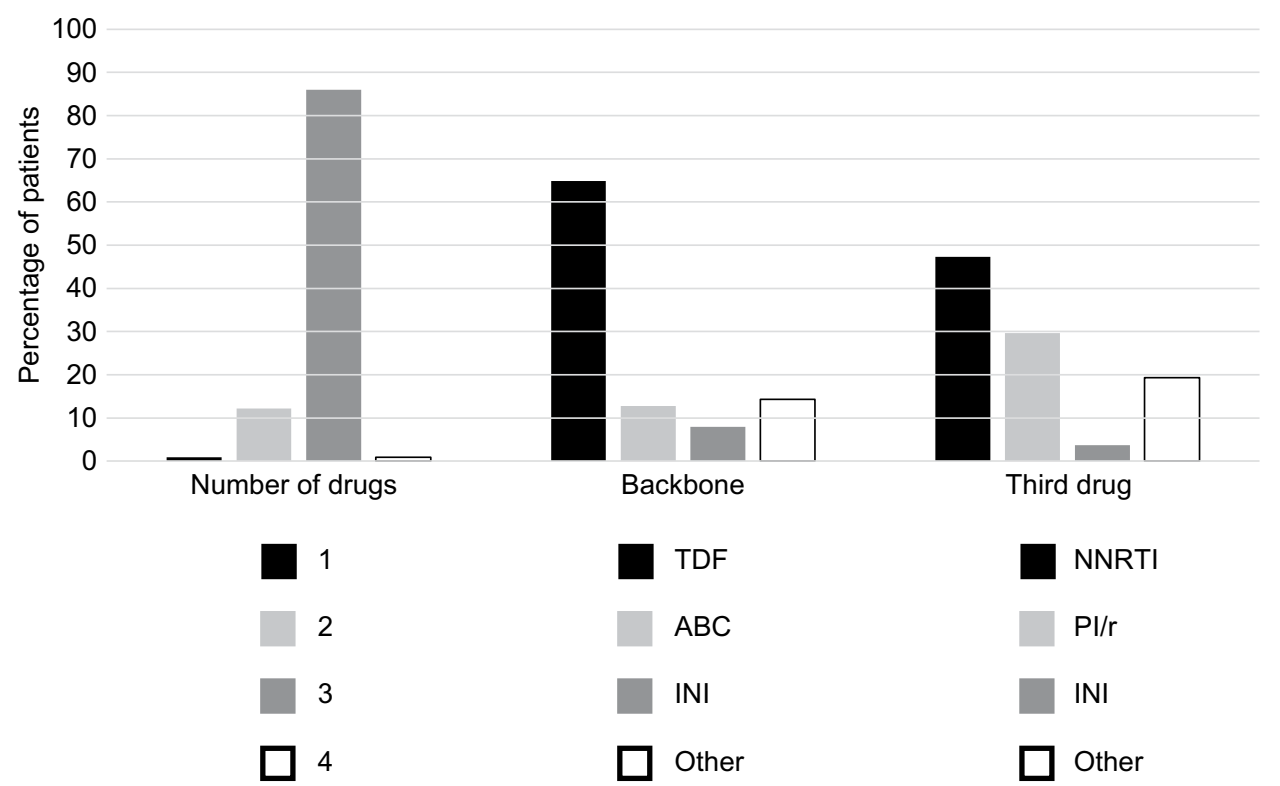

Figure 2 Use of antiretroviral drugs in the cohort.

Abbreviations: ABC, abacavir; INI, integrase inhibitor; NNRTI, non-nucleoside reverse transcriptase inhibitor; PI/r, protease inhibitor; TDF, tenofovir. 


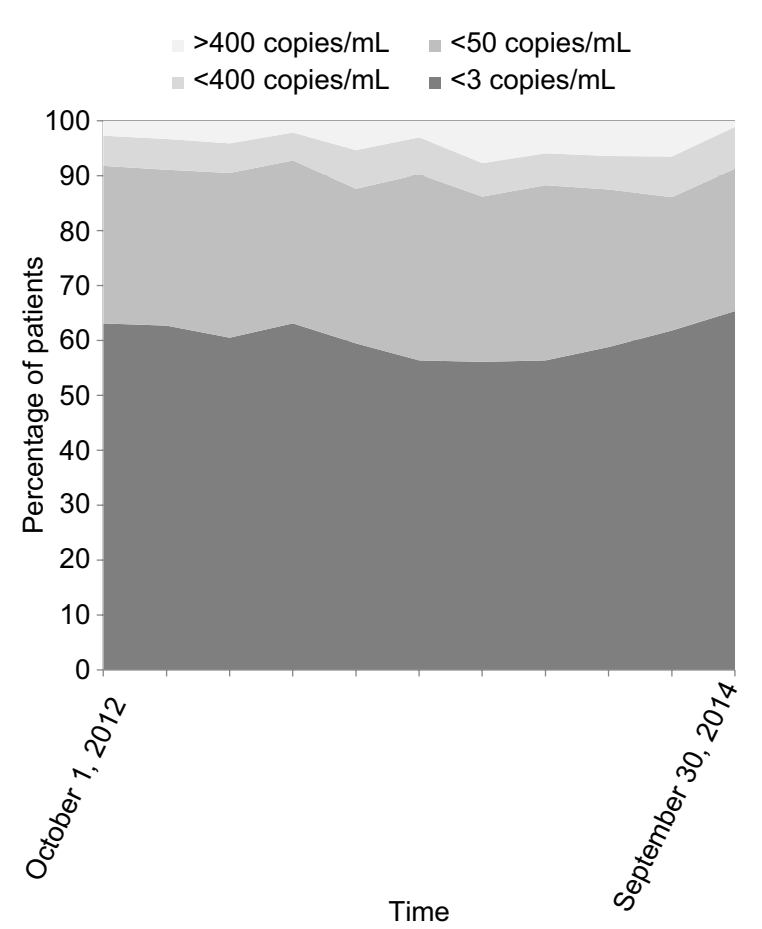

Figure 3 Virological response over time.

Note: Proportion of patients below a given threshold (HIV-RNA copies $/ \mathrm{mL}$ ).

In the multivariable model, several variables acted as possible predictors of virological outcome. These included total time on cART $(P=0.001)$ and time on current cART $(P=0.003)$ being longer times associated to a better outcome, third drug class $(P=0.007)$ being NNRTIs associated to a better outcome compared with PIs and adherence ( $P=0.018)$.

Mean adherence was high ( $91.4 \%$; $95 \%$ CI from $90.6 \%$ to $92.1 \%$ ) with $50 \%$ of patients showing an adherence rate $\geq 95 \%$. Variables associated with this parameter included transmission risk group $(P=0.035)$, ethnicity $(P<0.0001)$, age $(P=0.009)$, total time on cART $(P=0.002)$, and time on current $\operatorname{cART}(P=0.001)$. Adherence rate did correlate with the virological outcome. The mean adherence rate was $92.1 \%$ (95\% CI from $91.1 \%$ to $93.1 \%$ ) in $\mathrm{K}<3$ patients and was similar in $\mathrm{V}<3$ patients $(91.9 \%$; $95 \%$ CI from $91.0 \%$ to $92.8 \%$ ), but lowered to $88.8 \%$ (95\% CI from $86.2 \%$ to $91.5 \%$ ) in $\mathrm{K}>3$ patients and to $88.4 \%$ (95\% CI from $86.7 \%$ to $90.0 \%$ ) in $\mathrm{V}>50$ patients $(P<0.0001)$. A total of $55 \%$ of patients in group $\mathrm{K}<3$ and $\mathrm{V}<3$ showed an adherence rate $\geq 95 \%$, this proportion lowered to $51 \%$ in $\mathrm{K}>3$ patients and to $48 \%$ in $\mathrm{V}>50$ patients (Figure 4). Moreover, the greater proportion (34\%) of patients with a steady adherence rate $<95 \%$ fell in the $\mathrm{K}>3$ group, while most of those with an adherence rate $<95 \%$ because of a drug holiday $(21.7 \%)$ were observed in the $\mathrm{V}>50$ group ( $P=0.002$; Figure 4$)$.

\section{Discussion}

A residual LLV can still be detected in a vast proportion of individuals even after many years of suppressive cART. ${ }^{12,13}$ A number of studies have addressed the hypothetical association between residual LLV and negative virological outcomes, compared with those of patients with undetectable HIV-RNA using real-time assays that may detect HIV-RNA levels between 1 and 49 copies/mL..$^{11,14-18}$ The results have been inconclusive, if not contradictory, irrespective of the longitudinal or cross-sectional designs of studies, of the type or brand of assays, and of definitions of LLV (one, two, or several positive tests), and of the negative outcome considered. Therefore, some controversy remains over whether residual LLV is representative of viral release from the latent reservoir or is effectively a measure of ongoing viral replication.

The results of our study suggest that both factors may contribute to residual LLV. In previous studies on residual

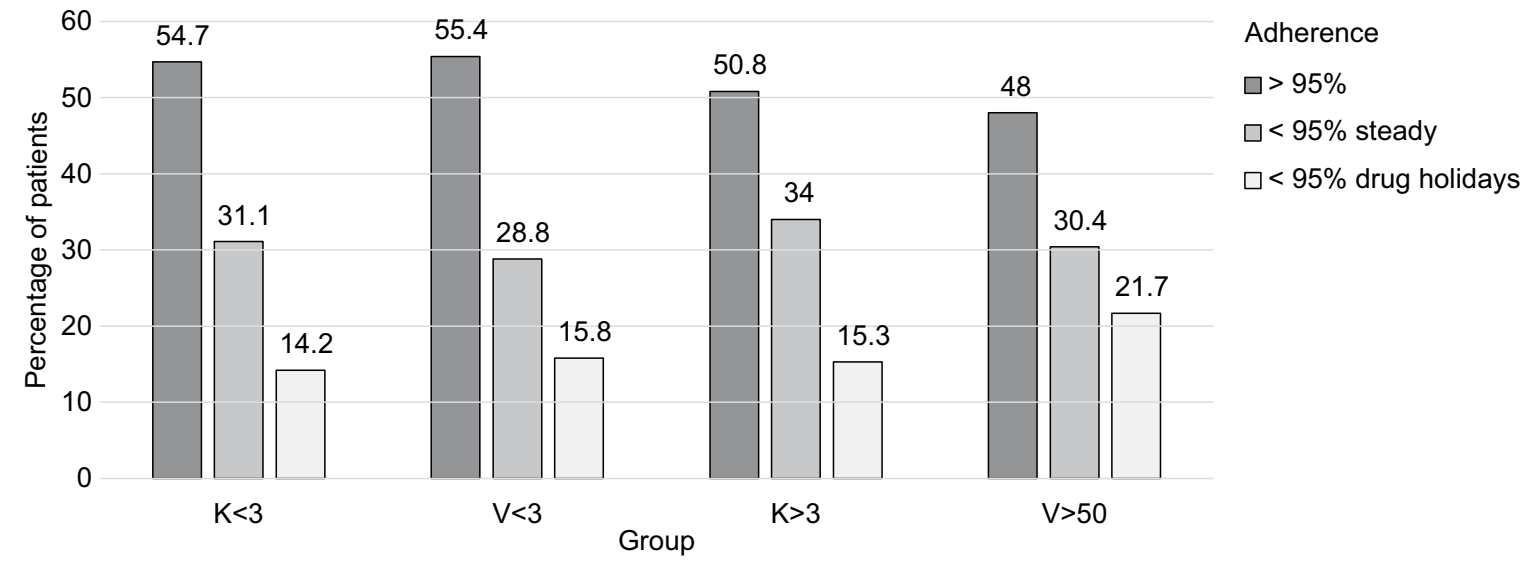

Figure 4 Type of adherence and virological response.

Notes: Patients were grouped according to HIV-RNA over time as $\mathrm{K}<3$ : constantly $<3$ copies $/ \mathrm{mL}$; $\mathrm{V}<3$ : sometimes below or above the cutoff limit but always $<50$ copies/mL; $K>3$ : constantly between 3 and 50 copies $/ \mathrm{mL}$; and $V>50$ : a measure of $>50$ copies $/ \mathrm{mL}$ minimum. 
HIV viremia in cART-treated individuals, cART adherence has been rarely assessed.

Apparently, there is no single accepted gold standard to measure adherence. ${ }^{19-21}$ Possible tools to measure adherence to cART include adherence diaries and self-report questionnaires, pill count, pharmacy refill records, and the Medication Event Monitoring System. 3,8,9,22

Each of these methods is associated with specific strengths and drawbacks. Self-reported adherence, although consistently associated with HIV-RNA response, generally shows a modest at the best relationship with the virological outcome and lacks of accuracy and precision. Self-reported adherence usually overestimates adherence because of recall bias and social desirability, often leading to the need to dichotomize the highly skewed data with cutoff points of $90 \%-100 \%$.

Manual pill count is a cheap and easy method to estimate adherence. However, it is time-consuming, requires a large staff, and does not assess pill sharing or dumping by the patients.

Pharmacy refill is another objective method that has higher potential. It can be easily applied in the clinical care setting, it is not negatively influenced by the recall bias and it does not require intensive need of staff. Pharmacy refill has been validated as a measure of cART adherence related to virological outcomes. ${ }^{22}$

We used pharmacy refill as a more objective, although proxy, indicator of adherence and extended it on a long period of time to capture variations in adherence rates or repeated behaviors.

Our finding is that a constant low-level detectable residual viremia is present in $50 \%$ of the patients, despite optimal (>95\%) cART adherence is consistent with the hypothesis that residual LLV depends on ongoing release of HIV from chronically infected cells (the latent reservoir). It supports previously reported evidence indicating minimal HIV evolution in patients with detectable residual LLV, homology of viral sequences in plasma and in latent virus from resting CD4 cells, and the absence of virological effect following cART intensification ${ }^{23-28}$ However, it is possible that in the remaining $50 \%$ of patients with incomplete cART adherence, we observed that viral replication contributes to residual LLV. These findings provide a potential explanation for some of the contradictory results previously reported such as evidence of ongoing viral evolution, changes in the viral reservoir after treatment intensification, or the association of residual viremia with risk of subsequent virological failure. ${ }^{11,15,29-32}$

These data are in accordance with recent studies that demonstrated how reduced cART adherence is associated with increased levels of HIV-RNA both in cells ${ }^{33}$ and plasma. ${ }^{34}$
Furthermore, we demonstrated that in patients with suboptimal adherence, the type of behavior may induce different virological outcomes. Participants with a steady adherence rate $<95 \%$ were more prone to present a constant measurable value of residual viremia within the limit of 50 copies $/ \mathrm{mL}$, while patients whose overall adherence rate was sub-optimal because of selective periods of reduced adherence (drug holidays), more frequently showed viral blips above the 50 copies threshold.

Together, these findings suggest that when incomplete cART adherence is present, a component of the plasma residual viremia may be due to new rounds of HIV replication. Such replication may further favor the persistence of HIV through the intermittent reseeding of the viral reservoir because of incomplete adherence, even in individuals with apparently successful cART and viral suppression when measured by commercial assays.

We are aware of some limitations of our study. They include the number of ARV regimens in a real-world cohort and the possible use of older ARV regimens in a subset of patients. Furthermore, some of the results (e.g., the relationship between drug classes and residual viremia and/or drug classes and adherence) may suffer of channeling biases. However, these limits may only influence the magnitude of the effect and not the effect itself. The association of residual LLV with cART adherence was still evident even after controlling for cART regimens. Another possible limit is the fact that incompletely adherent individuals may have been tested for HIV-RNA less frequently. Missing eventual LLV values, however, would have rather led to underestimate the role of reduced adherence and would not negatively influence the overall result. A further limit could be the relatively high proportion of undetectable patients we found as this may complicate the statistical analysis. Other parameters previously associated with LLV such as nadir CD4 counts or the amount of viral reservoir were not analyzed since these parameters were not available. ${ }^{31,32}$

Finally, therapeutic drug monitoring not being a standard of care, we cannot rule out a possible effect due to incomplete drug absorption or inadequate distribution of drugs into some compartments.

\section{Conclusion}

A steady viral suppression can occur despite moderate cART non-adherence, but such reduced adherence is often associated with low-level residual viremia, which could reflect new rounds of HIV replication. However, a detectable HIV-RNA can also be detected in patients with optimal cART adherence, indicating additional mechanisms favoring HIV persistence.

Our results suggest a benefit of routine medication adherence counseling to counter the effects of treatment fatigue 
in patients on long-term cART with apparently successful virological suppression.

\section{Disclosure}

FM has served as a consultant on advisory boards for Boehringer Ingelheim, Bristol-Myers Squibb, Gilead, GlaxoSmithKline, and Tibotec; he has received lecture fees from Bristol-Myers Squibb, Gilead, GlaxoSmithKline, and Merck Sharp \& Dohme, and has received research and educational grants from Boehringer Ingelheim, Bristol-Myers Squibb, GlaxoSmithKline, Jansen-Cilag, and Roche. The other authors report no conflicts of interest in this work.

\section{References}

1. Antiretroviral Therapy Cohort Collaboration, Zwahlen M, Harris R, May $\mathrm{M}$, et al. Mortality of HIV-infected patients starting potent antiretroviral therapy: comparison with the general population in nine industrialized countries. Int J Epidemiol. 2009;38(6):1624-1633.

2. Wood E, Hogg RS, Yip B, Harrigan PR, Montaner JS. Why are baseline HIV RNA levels 100,000 copies/mL or greater associated with mortality after the initiation of antiretroviral therapy? J Acquir Immune Defic Syndr. 2005;38(3):289-295.

3. Maggiolo F, Airoldi M, Kleinloog HD, et al. Effect of adherence to HAART on virologic outcome and on the selection of resistance-conferring mutations in NNRTI- or PI-treated patients. HIV Clin Trials. 2007; 8(5):282-292.

4. Guidelines for treatment of HIV-positive adults in Europe. Guidelines Chair and Coordinator: Lundgren JD. Available from: http://www. eacsociety.org/files/guidelines_8.0-english-revised_2016-0610.pdf. Accessed April 12, 2017.

5. Guidelines for the Use of Antiretroviral Agents in HIV-1-Infected Adults and Adolescents. Chair and Coordinator: Churchill D. Available from: http://www.bhiva.org/documents/Guidelines/Treatment/2015/2015treatment-guidelines.pdf. Accessed April 12, 2017.

6. Labbett W, Garcia-Diaz A, Fox Z, et al. Comparative evaluation of the ExaVirTM Load version 3 reverse transcriptase assay for the measurement of HIV-1 plasma viral load. J Clin Microbiol. 2009;47(10):3266-3270.

7. Gandhi RT, Deeks SG. Plasma HIV-1 RNA levels during antiretroviral therapy: how low is low enough? Clin Infect Dis. 2012;54(5):733-735.

8. Goldman JD, Cantrell RA, Mulenga LB, et al. Simple adherence assessments to predict virologic failure among HIV-infected adults with discordant immunologic and clinical responses to antiretroviral therapy. AIDS Res Hum Retroviruses. 2008;24(8):1031-1035.

9. Fairley CK, Permana A, Read TRH. Long-term utility of measuring adherence by self-report compared with pharmacy record in a routine clinic setting. HIV Med. 2005;6(5):366-369.

10. Wood E, Hogg RS, Yip B, Harrigan PR, O'Shaughnessy MV, Montaner JS. Effect of medication adherence on survival of HIV-infected adults who start highly active antiretroviral therapy when the CD4+ cell count is 0.200 to $0.350 \times 10(9)$ cells/L. Ann Intern Med. $2003 ; 139(10)$ : $810-816$.

11. Maggiolo F, Callegaro A, Cologni G, et al. Ultrasensitive assessment of residual low-level HIV viremia in HAART treated patients and risk of virologic failure. J Acquir Immune Defic Syndr. 2012;60(5): 473-482.

12. Palmer S, Maldarelli F, Wiegand A, et al. Low-level viremia persists for at least 7 years in patients on suppressive antiretroviral therapy. Proc Natl Acad Sci U S A. 2008;105(10):3879-3884.

13. Hatano H, Delwart EL, Norris PJ, et al. Evidence of persistent low-level viremia in long-term HAART-suppressed, HIV-infected individuals. AIDS. 2010;24(16):2535-2539.
14. Havlir DV, Koelsch KK, Strain MC et al; Gilead 903 Study Team. Predictors of residual viremia in HIV-infected patients successfully treated with efavirenz and lamivudine plus either tenofovir or stavudine. J Infect Dis. 2005;191(7):1164-1168.

15. Doyle T, Smith C, Vitiello P, et al. Plasma HIV-1 RNA detection below 50 copies $/ \mathrm{mL}$ and risk of virologic rebound in patients receiving highly active antiretroviral therapy. Clin Infect Dis. 2012;54(5):724-732.

16. Charpentier C, Landman R, Laouenan C, et al. Persistent low-level HIV-1 RNA between 20 and 50 copies/mL in antiretroviral-treated patients: associated factors and virological outcomes. J Antimicrob Chemother. 2012;67(9):2231-2235.

17. Henrich TJ, Wood BR, Kuritzkes DR. Increased risk of virological rebound in patients with antiviral therapy with a detectable HIV load $<48$ copies/mL. PLoS One. 2012;7(11):e50065.

18. Gianotti N, Galli L, Salpietro S, et al. Virological rebound in human immunodeficiency virus-infected patients with or without residual viraemia: results from an extended follow-up. Clin Microbiol Infect. 2013; 19(12):E542-E544.

19. Berg KM, Arnsten JH. Practical and conceptual challenges in measuring antiretroviral adherence. J Acquir Immune Defic Syndr. 2006; 43(Suppl 1):S79-S87.

20. Hess LM, Raebel MA, Conner DA, Malone DC. Measurement of adherence in pharmacy administrative databases: a proposal for standard definitions and preferred measures. Ann Pharmacother. 2006;40(7-8):1280-1288.

21. Deschamps AE, De Geest S, Vandamme AM, Bobbaers H, Peetermans WE, Van Wijngaerden E. Diagnostic value of different adherence measures using electronic monitoring and virologic failure as reference standards. AIDS Patient Care STDS. 2008;22(9):735-743.

22. Grossberg R, Zhang Y, Gross R. A time-to-prescription-refill measure of antiretroviral adherence predicted changes in viral load in HIV. J Clin Epidemiol. 2004;57(10):1107-1110.

23. Hermankova M, Ray SC, Ruff C, et al. HIV-1 drug resistance profiles in children and adults with viral load of $<50$ copies $/ \mathrm{mL}$ receiving combination therapy. JAMA. 2001;286(2):196-207.

24. Bailey JR, Sedaghat AR, Kieffer T, et al. Residual human immunodeficiency virus type 1 viremia in some patients on antiretroviral therapy is dominated by a small number of invariant clones rarely found in circulating CD4+ T cells. J Virol. 2006;80(13):6441-6457.

25. Anderson JA, Archin NM, Ince W, et al. Clonal sequences recovered from plasma from patients with residual HIV-1 viremia and on intensified antiretroviral therapy are identical to replicating viral RNAs recovered from circulating resting CD4+ T cells. JVirol. 2011;85(10):5220-5223.

26. McMahon D, Jones J, Wiegand A, et al. Short-course raltegravir intensification does not reduce persistent low-level viremia in patients with HIV-1 suppression during receipt of combination antiretroviral therapy. Clin Infect Dis. 2010;50(6):912-919.

27. Gandhi RT, Zheng L, Bosch RJ, et al; AIDS Clinical Trials Group A5244 team. The effect of raltegravir intensification on low-level residual viremia in HIV-infected patients on antiretroviral therapy: a randomized controlled trial. PLoS Med. 2010;7(8):e1000321.

28. Llibre JM, Buzon MJ, Massanella M, et al. Treatment intensification with raltegravir in subjects with sustained HIV-1 viraemia suppression: a randomized 48-week study. Antivir Ther. 2012;17(2):355-364.

29. Zhang L, Ramratnam B, Tenner-Racz K, et al. Quantifying residual HIV-1 replication in patients receiving combination antiretroviral therapy. N Engl J Med. 1999;340(21):1605-1613.

30. Calcagno A, Motta I, Ghisetti V, et al. HIV-1 very low level viremia is associated with virological failure in highly active antiretroviral treatmenttreated patients. AIDS Res Hum Retroviruses. 2015;31(10):999-1008.

31. Havlir DV, Strain MC, Clerici M, et al. Productive infection maintains a dynamic steady state of residual viremia in human immunodeficiency virus type 1-infected persons treated with suppressive antiretroviral therapy for five years. J Virol. 2003;77(20):11212-11219.

32. Ramratnam B, Ribeiro R, He T, et al. Intensification of antiretroviral therapy accelerates the decay of the HIV-1 latent reservoir and decreases, but does not eliminate, ongoing virus replication. J Acquir Immune Defic Syndr. 2004;35(1):33-37. 
33. Pasternak AO, de Bruin M, Jurriaans S, et al. Modest nonadherence to antiretroviral therapy promotes residual HIV-1 replication in the absence of virological rebound in plasma. J Infect Dis. 2012;206(9):1443-1452.
34. Li JZ, Gallien S, Ribaudo H, Heisey A, Bangsberg DR, Kuritzkes DR. Incomplete adherence to antiretroviral therapy is associated with higher levels of residual HIV-1 viremia. AIDS. 2014;28:181-186.

\section{Publish your work in this journal}

Pragmatic and Observational Research is an international, peer-reviewed open access journal that publishes data from studies designed to reflect more closely medical interventions in real-world clinical practice compared with classical randomized controlled trials (RCTs). The manuscript management system is completely online and includes a very quick and fair peer-review

\section{Dovepress}

system. Visit http://www.dovepress.com/testimonials.php to read real quotes from published authors. 